\title{
Simulation of ion beam sputtering with SDTrimSP, TRIDYN and SRIM
}

H. Hofsäss ${ }^{\text {a) }}$, K. Zhang ${ }^{\text {a) }}$, A. Mutzke ${ }^{\text {b) }}$

a) $2^{\text {nd }}$ Institute of Physics, University of Göttingen, Friedrich-Hund-Platz 1, 37077 Göttingen, Germany

b) Max-Planck Institute for Plasma Physics, Wendelsteinstrasse 1, 17491 Greifswald, Germany

corresponding author: H. Hofsäss, hans.hofsaess@phys.uni-goettingen.de , +49551397669

\begin{abstract}
A quantitative simulation of ion beam sputtering and related collision cascade effects is essential for applications of ion beam irradiation in thin film deposition, surface treatment and sculpting with focused ion beams, ion beam smoothing of surfaces and ion-induced nanopattern formation. The understanding of fundamental ion-solid interaction processes relevant for nanostructure formation, ion-induced mass redistribution, sputter yield amplification, ion beam mixing and dynamic compositional changes requires reliable simulations of ion-solid interaction processes in particular at low ion energies.

In this contribution we discuss the possibilities, the key benefits and the limitations of three popular binary collision Monto Carlo simulation programs (SDTrimSP, TRIDYN and SRIM). The focus will be set to the calculation of angle dependent sputter yields, angular distribution of sputtered particles, sputter yields for compound materials, sputter yield amplification effects, as well as the extraction of parameters relevant for modelling ion-induced surface pattern formation from vacancy and recoil atom distributions.
\end{abstract}

Keywords: ion sputtering, binary collision simulation, SDTrimSP, TRIDYN, SRIM 


\section{Introduction}

The erosion of atoms from a solid surface by bombardment of energetic particles is known as sputtering and has been subject of intense research for more than 50 years $[1,2]$. Several comprehensive reviews on all aspects of sputtering [3,4,5,6,7] and applications of sputtering to low energy ion deposition on surfaces [8] or secondary ion mass spectrometry [9] were published to date. Theoretical models of sputtering were able to describe the main features of the sputter process, such as the sputter yield (removed number of atoms from a surface per incident ion), the correlation of the sputtering yield with the nuclear energy loss, the dependence of the sputtering yield on projectile incidence angle, projectile species and projectile and target mass and the overall angular emission distribution of sputtered atoms $[1,2,10]$.

Research on sputtering and its application was accompanied by the development of computer simulation codes mainly based on Monte Carlo simulations of binary collision processes [11-15]. Today the most spread simulation software is SRIM [16], because of its convenient user interface and its extensive database on compound target materials and electronic energy loss data. It is based on the TRIM code [17] and uses the ZBL universal interaction potential [17] and Biersack’s magic formula [18] to solve the scattering integral. TRIDYN and SDTrimSP are similar to TRIM but use the Krypton-Carbon interaction potential [19] and allows dynamic simulations, taking care of stoichiometry changes due to incorporation of projectile atoms, atomic mixing, as well as preferential sputtering [20]. The SDTrimSP code (SD = static-dynamic; SP = sequential and parallel processing) is a further development of TRIDYN with focus on low energy collisions and sputter processes [21,22]. The physics behind SDTrimSP is described in the book Computer simulation of ion-solid interaction by W. Eckstein [14]. SDTrimSP comes with a large variety of input options, including the choice of different interaction potentials and different integration methods for 
the scattering integral. Furthermore SDtrimSP produces a multiplicity of output data, including all details of sputtered atoms.

Simulations were compared with numerous experimental sputter yield data (e.g. refs $[23,24])$ and often reasonable quantitative agreement is obtained. A less comprehensive comparison with experimental data exists on the angular distributions of sputtered atoms and the sputtering yield dependence on ion incidence angle [23,25-33].

In this contribution we compare results of sputter simulations using SRIM, TRIDYN and SDTrimsP for several selected cases and also compare with experimental data.

\section{Experimental}

Angular dependent sputter yields were measured for $1 \mathrm{keV}$ Xe ion irradiation of Si and Ge, $10 \mathrm{keV}$ Xe ion irradiation of $\mathrm{Si}$ [30] as well as $5 \mathrm{keV}$ Xe ion irradiation of a Fe film deposited on Si. For irradiations with $\mathrm{Xe}$ ions at $5 \mathrm{keV}$ or $10 \mathrm{keV}$ ion energy we used a mass selected Colutron ${ }^{\circledR}$ ion beam system with Wien-filter for mass selection and a beam sweep to obtain a uniform exposure over an area of about $10 \mathrm{~mm}$ in diameter with angular spread below $1^{\circ}$ [34]. Ion irradiations were done at room temperature. The pressure during erosion was $2 \times 10^{-6} \mathrm{~Pa}$ and the ion flux was about $1-2 \mu \mathrm{A} / \mathrm{cm}^{2}$. Low energy Xe irradiations were done using a microwave plasma ion source (Tectra Gen II) with a broad beam and substrates positioned at about $40 \mathrm{~cm}$ distance. The sample holder was water-cooled during irradiation. The ion flux was kept between $2-5 \times 10^{14}$ ions $/ \mathrm{cm}^{2} / \mathrm{s}\left(30-60 \mu \mathrm{A} / \mathrm{cm}^{2}\right)$ and measured with a Faraday cup, which could be placed in front of the sample holder.

To determine the erosion depth for $\mathrm{Si}$ and Ge, part of the substrate was covered with a Si wafer and the erosion depth was measured using a DECTAC® profilometer. The erosion depth for the Fe film was determined from the residual film thickness measured using Rutherford-Backscattering with $900 \mathrm{keV} \mathrm{He}{ }^{2+}$ ions. 


\section{Simulation details}

Simulations of collision cascades, sputtering yields as well as angular distributions of sputtered atoms were done using the binary collision Monte Carlo programs SDTrimSP V5.0 [22] and SRIM Version 2008 and 2013 [16]. An important input parameter for sputter yield simulations is the surface binding energy. The surface binding energy is derived form the standard formation enthalpy of the respective element. All three programs use the same values, tabulated for all possible target elements. For the case of a gaseous component, the tabulated elemental surface binding energy corresponds to the atomic fraction of the molecular dissociation energy. The TRIDYN manual [35] recommends the use of different surface binding energies in the case of compound materials. Here, essentially the formation enthalpy of the compound is added to the to the elemental surface binding energy. However, this leads to significantly high surface binding ene rgies $_{\text {and }}$ thus to very low sputter yields, compared to experimental data in the case of $\mathrm{SiO}^{2}$ and $\mathrm{Ta}_{2} \mathrm{O}_{5}$ discussed in this contribution. Therefore we use the tabulated elemental surface binding energies for all simulations.

SRIM uses default values for the displacement energy $E_{D}$ (typically $25 \mathrm{eV}$ ) and the bulk binding energy (typically $3 \mathrm{eV}$ ). In contrast, the TRIDYN manual recommends a value of $\mathrm{ED} \sim 8 \mathrm{eV}$ for amorphous materials and $\mathrm{E}_{\mathrm{B}}=0$. Strictly, the displacement energy is only defined in crystalline materials. Here, recoils with recoil kinetic energy below the displacement threshold energy are reset to their original position, if they come to rest within the target. For sputtered recoils, the displacement threshold is not relevant. Displacement energies are essentially experimental data obtained from electron irradiation experiments [8,36,37]. The displacement energy is hardly defined for amorphous materials and almost impossible to verify experimentally. Therefore, for amorphous materials it is meaningful to use small or even zero displacement energies. The choice of $E_{D}$ influences the calculated atomic mass transport within a collision cacade and atomic mixing. For example, in a low ion energy collision cascade calculated with large $E_{D}$ values, most generated recoils are reset to 
their original position (resulting in zero average mass transport) and a only few recoils with large displacement distances remain. The bulk binding energy is the energy required to create a vacancy in a crystalline structure. Again, this energy is hardly defined in an amorphous material, so that it is recommended to use a zero bulk binding energy. In TRIDYN and SDTrimSP one must reduce the surface binding energy correspondingly, if a bulk binding energy $>0$ is chosen. Therefore the bulk binding energy has usually little influence on the sputtering yield.

The screening functions of the $\mathrm{KrC}$ and ZBL interaction potentials (see chapter 4.1 of ref [14]) are quite similar for a reduced interaction radius $r / a<<30$ (internuclear separation $<<2 \AA$ ) with screening length a (see Fig. 4.1 of ref [14]). However, the screening for larger $\mathrm{r} / \mathrm{a}$ is much stronger for the $\mathrm{KrC}$ potential, which seems to be benefitial in particular for simulations of low energy collisions with larger distances between two atoms involved in a scattering process. The screening function of the $\mathrm{ZBL}$ and $\mathrm{KrC}$ potentials for interaction of $\mathrm{Si}$ and $\mathrm{W}$ atoms are plotted in Fig. 1. The screening is significantly stronger for the $\mathrm{KrC}$ potential in particular if one of the atoms has a high atomic number (see also Fig. 4.4 in ref. [14], which shows the screening functions for Au-Au interactions).

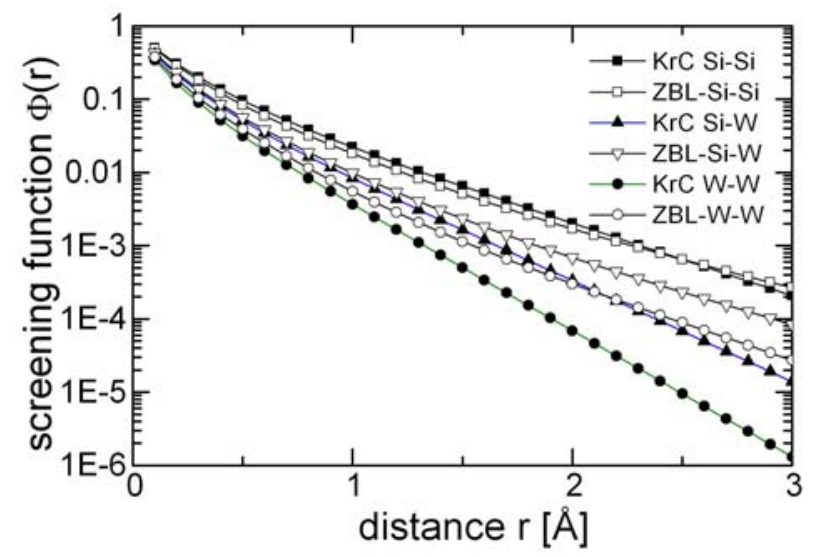

Figure 1: Screening function used in the $\mathrm{KrC}$ and $\mathrm{ZBL}$ potential for Si-Si, Si-W and W-W scattering. 


\section{Results and discussion}

\subsection{Ion incidence angle dependence of the sputter yield}

In Fig. 2 we compare the measured sputter yield as function of ion incidence angle for $1 \mathrm{keV}$ Xe ion irradiation of Si and Ge with SRIM and SDTrimSP simulations. Whereas SDTrimSP is in good quantitative agreement with the experimental data, SRIM sputter yields strongly deviate in particular at large angles of incidence. Furthermore, the zero degree sputter yield for Ge is almost a factor of 2 smaller compared to the SDTrimSP simulation and the experimental sputter yield. This underestimation of the sputter yield by SRIM seems to be systematic for heavy projectiles and lighter target elements. Some examples are listed in Table I. Significant deviations between SRIM sputter yields and experimental data were also found for $10 \mathrm{keV}$ Xe ion irradiation of Si as function of ion incidence angle [30]. Again, SDTrimSP gave good quantitative agreement with the experiment.

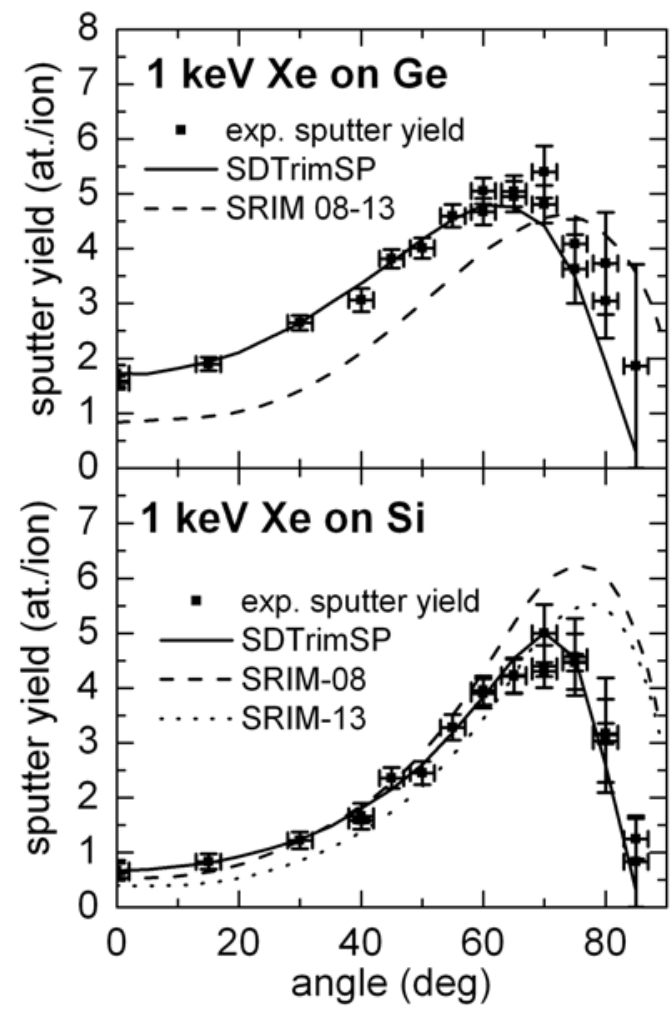

Figure 2: Experimental sputter yield as function of ion incidence angle for $1 \mathrm{keV}$ Xe on Si and Ge and values from simulations with SRIM and SDTrimSP. 


\begin{tabular}{|c|c|c|c|c|}
\hline & $\mathrm{M}_{\mathrm{P}} / \mathrm{M}_{\mathrm{T}}$ & $\begin{array}{l}\text { SRIM-2013 } \\
\text { Y (atoms/ion) }\end{array}$ & $\begin{array}{c}\text { SDTrimSP } \\
\text { Y(atoms/ion) }\end{array}$ & $\begin{array}{c}\Delta \mathrm{Y} / \mathrm{Y}_{\text {SDtrimSP }} \\
(\%)\end{array}$ \\
\hline $1 \mathrm{keV}$ Ar on $\mathrm{Ge}$ & $<1$ & 1.84 & 1.88 & -2 \\
\hline $1 \mathrm{keV}$ Ar on Mo & $<1$ & 1.65 & 1.42 & +16 \\
\hline $1 \mathrm{keV}$ Ar on Ta & $<1$ & 1.54 & 1.15 & +34 \\
\hline $1 \mathrm{keV} \mathrm{Au}$ on $\mathrm{Ti}$ & $>1$ & 0.32 & 1.02 & -68 \\
\hline $1 \mathrm{keV}$ Ti on $\mathrm{Au}$ & $<1$ & 4.37 & 2.92 & +50 \\
\hline $1 \mathrm{keV}$ Xe on Ge & $>1$ & 0.87 & 1.73 & -50 \\
\hline $1 \mathrm{keV}$ Xe on Mo & $>1$ & 0.80 & 1.35 & -41 \\
\hline $1 \mathrm{keV}$ Xe on Ta & $<1$ & 1.82 & 1.45 & +25 \\
\hline $5 \mathrm{keV}$ Xe on Ge & $>1$ & 2.65 & 3.95 & -33 \\
\hline $5 \mathrm{keV}$ Xe on Mo & $>1$ & 2.50 & 3.48 & -28 \\
\hline $5 \mathrm{keV}$ Xe on Ta & $<1$ & 5.10 & 3.75 & +36 \\
\hline
\end{tabular}

Table I. Comparison of calculated sputter yields $Y$ for normal ion incidence. $M_{P} / M_{T}$ is the ratio of projectile mass and target mass. For $\mathrm{M}_{\mathrm{P}} / \mathrm{M}_{\mathrm{T}}>1\left(\mathrm{M}_{\mathrm{P}} / \mathrm{M}_{\mathrm{T}}<1\right)$ the SRIM sputter yields are significant smaller (larger) compared to the SDTrimSP sputter yields.

\subsection{Angular distributions of sputtered atoms}

The most striking differences between SRIM and SDTrimsP were found for the angular distributions $\mathrm{f}(\beta, \varphi)$ of sputtered atoms with polar emission angle $\beta$ and azimutal angle $\varphi$. For normal ion incidence we would expect a cosine distribution $f(\cos \beta)=f_{0} \cdot \cos \beta$ [38]. To plot the calculated angular distributions we choose a stereographic projection of the emission hemisphere with equidistant polar angle lines of latitude. In this way the emission distribution into the hemisphere is projected to a $2 \mathrm{D}$ matrix with each matrix element having the same solid angle $d \Omega$. Here we have $d \Omega=2.74 \cdot 10^{-3}$, corresponding to an angular segment of $3^{\circ} \times 3^{\circ}$. The projected direction of the incident ion beam points along the horizontal direction towards azimuthal angle $0^{\circ}$. The stereographic projection has the advantage that the angular emission distribution is visualized without significant distortion and deviations from a polar symmetric distribution, such as a forward directed distribution, can be easily seen. On the other hand, plotting the differential sputter yield as function of the cosine of the polar angle should give a 
linear dependence in case of a cosine distribution. From a cut through a stereographic projection we obtain 1-dimensional polar plots showing the emission distribution with respect to a given azimuthal direction.

As a first example we calculated the angular distribution for $2 \mathrm{keV}$ Cs ion irradiation of Si and Ge at incidence angles of $\theta=30^{\circ}, 45^{\circ}$ and $60^{\circ}$ and compare to existing experimental data [29]. The simulation results for ions incident at $\theta=60^{\circ}$ and about $10^{5}$ sputtered atoms are shown in Fig.3. The angular distributions of sputtered atoms is axially symmetric with respect to the surface normal, if calculated with SRIM-2013, and the 1-dimensional distribution has its maximum in direction of the surface normal (Fig. 3c,d). SDTrimSP calculates a broader distribution with a clear emission maximum in forward direction located around $\beta \approx 20^{\circ}-30^{\circ}$ for Si and about $ß \approx 15^{\circ}$ for Ge (Fig. 3a,b). The corresponding 1-dimensional polar plots of these distributions are in good quantitative agreement with the experimental result reported in [29].

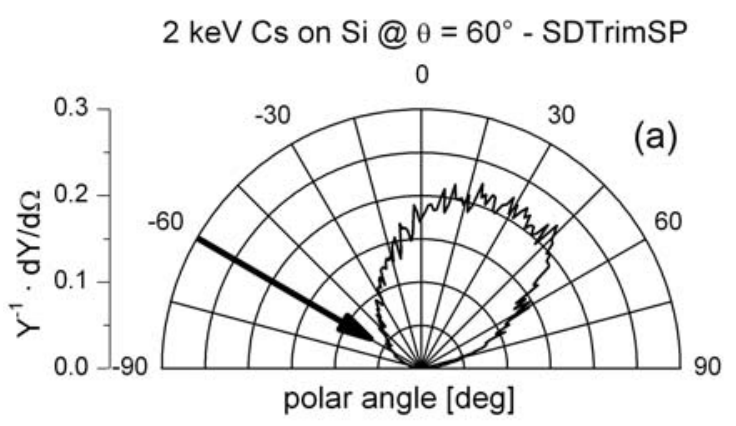

2 keV Cs on Si @ $\theta=60^{\circ}$ - SRIM2013

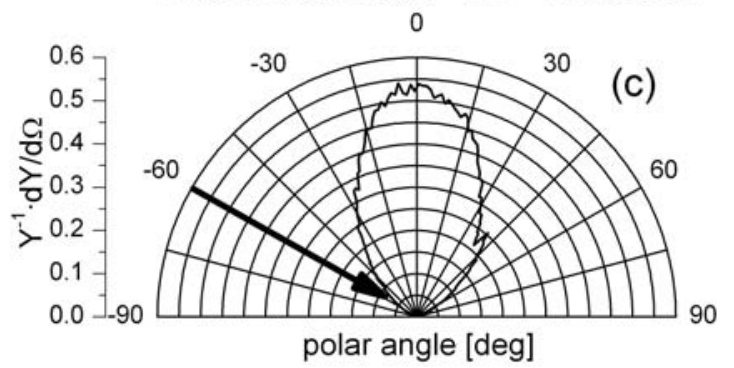

2 keV Cs on Ge @ $\theta=60^{\circ}$ - SDTrimSP

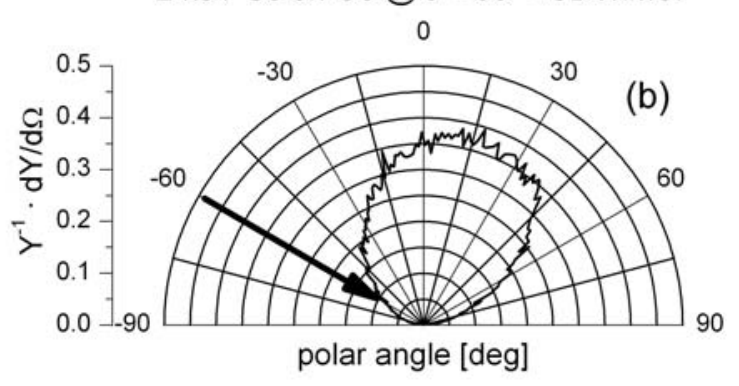

$2 \mathrm{keV}$ Cs on Ge @ $\theta=60^{\circ}$ - SRIM2013

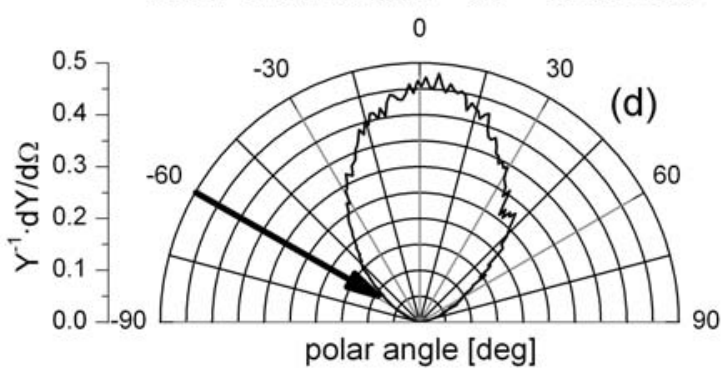

Figure 3: SDTrimSP and SRIM-2013 simulation of the angular distribution of sputtered Si and $\mathrm{Ge}$ atoms for ion irradiation of $\mathrm{Si}$ or $\mathrm{Ge}$ with $2 \mathrm{keV} \mathrm{Cs}$ ions incident at $\theta=60^{\circ}$, corresponding to the experimental data from ref. [29]. Upper row (a),(b): 1-dimensional polar plot of the emission distribution for Si and Ge calculated with SDTrimSP. Lower row (c),(d): 1-dimensional polar plot of the emission distribution for Si and Ge calculated with SRIM- 
2013.. The arrows indicate the direction of ion incidence. The gray scale represents the normalized sputter yield per solid angle $\mathrm{d} \Omega$.

Another comparison was done for $4 \mathrm{keV} \mathrm{D}^{+}$ions on $\mathrm{Ni}$, which was experimentally investigated by Becerra-Acevedo et al. [26]. The experimental angular distribution of sputtered atoms exhibits a forward emission of sputtered particles, including a pronounced feature generated by single knock-on collisions. Becerra-Acevedo et al. obtained good quantitative agreement using TRIM.SP simulations (a predecessor version of SDTrimSP). SDTrimSP exactly reproduces the simulations from 1984 (Fig. 4a), including the feature generated by single knock-on collisions. The forward emission is obvious from in Fig.4a because few sputtered atoms occur at azimuthal angles $>90^{\circ}$. In contrast, SRIM-2013 calculates a distribution with somewhat smaller polar angles, which is axially symmetric along the surface normal (Fig. 4b), i.e which has no dependence on the azimuthal angle. There is also the feature arising from single knock-on collisions visible, but at slightly smaller longitudinal polar angle compared to SDTrimSP.

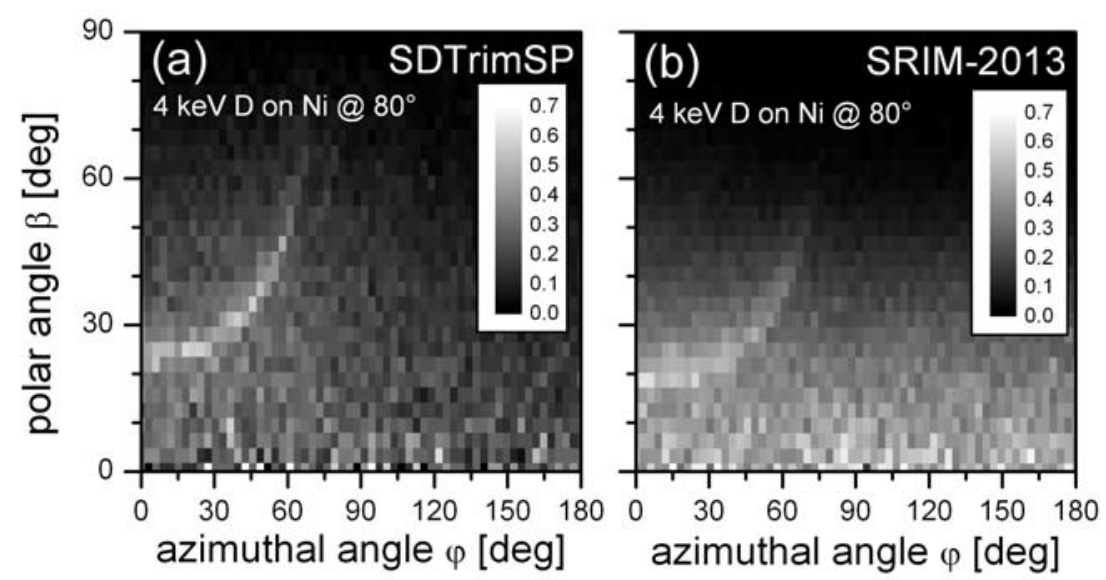

Figure 4: Simulation of the angular distribution of sputtered $\mathrm{Ni}$ atoms for $4 \mathrm{keV} \mathrm{D}^{+}$ion irradiation of $\mathrm{Ni}$ at $80^{\circ}$ ion incidence angle for about $10^{5}$ sputter atoms. left: plot of polar and azimuthal angle calculated with SDTrimSP similar to the plot shown in ref. [26]. Right: plot of polar and azimuthal angle calculated with SRIM-2013. The grayscales represent the normalized differential sputter yield $\mathrm{Y}^{-1} \cdot \mathrm{dY} / \mathrm{d} \Omega$. Both distributions show a pronounced contribution of single knock-on collisions, visible as brighter rim in the plots. In addition, SRIM-2013 shows a symmetric distribution of sputtered atoms around the surface normal, whereas SDtrimSP indicates forward directed sputtering. 
Surprisingly, if the simulations shown in Fig. 3c for the Si target are done with SRIM2008, the emission distribution is strongly peaked in surface normal direction, i.e. SRIM-2008 wrongly predicts, that almost all sputtered atoms would be emitted along the surface normal direction. To further evaluate this exotic angular emission distribution obtained with SRIM2008, we did some simulations with SRIM-2013 for Si, Al and also targets containing light ions with $\mathrm{Z}_{\text {target }}<15$. In Fig. 5 we compare the angular distributions for $1 \mathrm{keV}$ Ar on $\mathrm{Si}$ calculated with SRIM-2008, SRIM-2013 and SDtrimSP. Indeed, SRIM-2008 produces a distribution which is strongly peaked along the surface normal for both incidence angles of $\theta$ $=0^{\circ}$ and $\theta=40^{\circ}$. Nearly all sputtered atoms would be emitted with polar angles $<3^{\circ}$. SRIM2013 creates a symmetric broader distribution for both incidence angles. However, the distribution strongly deviates from a cosine distribution. The angular distribution from SDTrimSP is an almost perfect cosine distribution and is a symmetric distribution around the surface normal for $\theta=0^{\circ}$ incidence angle and a clearly forward directed distribution for $\theta=$ $40^{\circ}$ incidence angle.
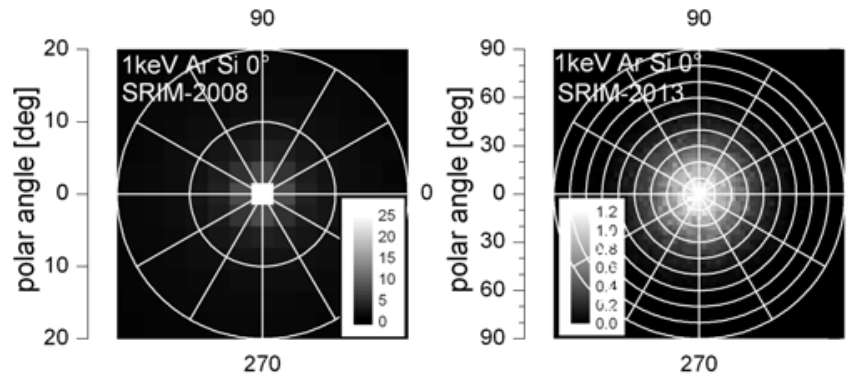

270

90

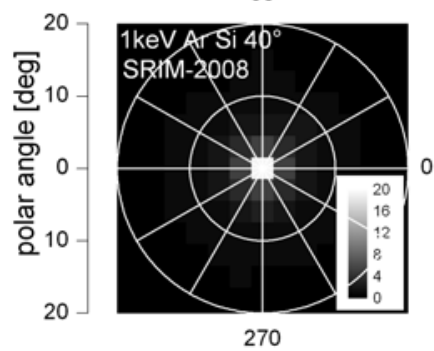

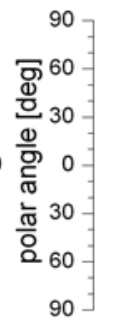

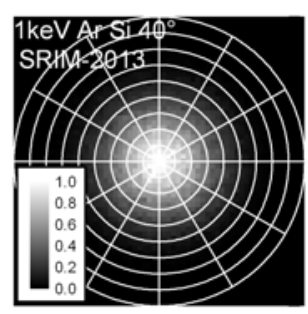

270

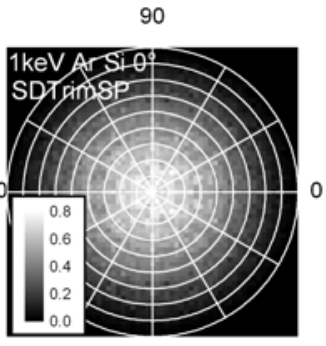

270

90

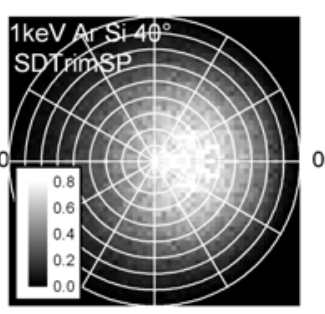

270

Figure 5: Calculated angular distributions of sputtered Si atoms for $1 \mathrm{keV}$ Ar ions on Si and ion incidence angles of $\theta=0^{\circ}$ and $\theta=40^{\circ}$. Left: calculation with SRIM-2008 (shown is the regime for polar angles $\beta<20^{\circ}$ ). Middle: calculation with SRIM-2013. Right: calculation with SDTrimSP. The gray scale corresponds to the normalized differential sputter yield $\mathrm{Y}^{-1} \cdot \mathrm{dy} / \mathrm{d}(\cos \theta)$. The azimuthal angles $\varphi=0^{\circ}, 90^{\circ}$ and $270^{\circ}$ are indicated. 
We now may study the SRIM-2013 distributions for various target atomic numbers and find that $\mathrm{Z}_{\text {target }}=14$ is a critical value. Fig. 6 shows the differential sputter yield as function of the cosine of the polar emission angle obtained for $1 \mathrm{keV}$ Ar on $\mathrm{Al}(\mathrm{Z}=13)$ calculated with SDTrimSP and SRIM-2013. SDTrimSP yields cosine distributions, except for $80^{\circ}$ ion incidence where we obtain a forward directed emission. From SRIM-2013 we obtain an emission distribution which is strongly peaked along surface normal direction, independent of the ion incidence angle. Such unusual distributions are always obtained from SRIM if the target contains light target element with $\mathrm{Z}_{\text {Target }}<14$ (Si). Therefore sputter data from SRIM2013 are wrong e.g. for $\mathrm{Na}, \mathrm{Mg}, \mathrm{Al}$ and all oxide, carbide, nitride or fluoride targets. For SRIM-2008 the error occurs for $\mathrm{Z}_{\text {Target }}<18$ (Ar).

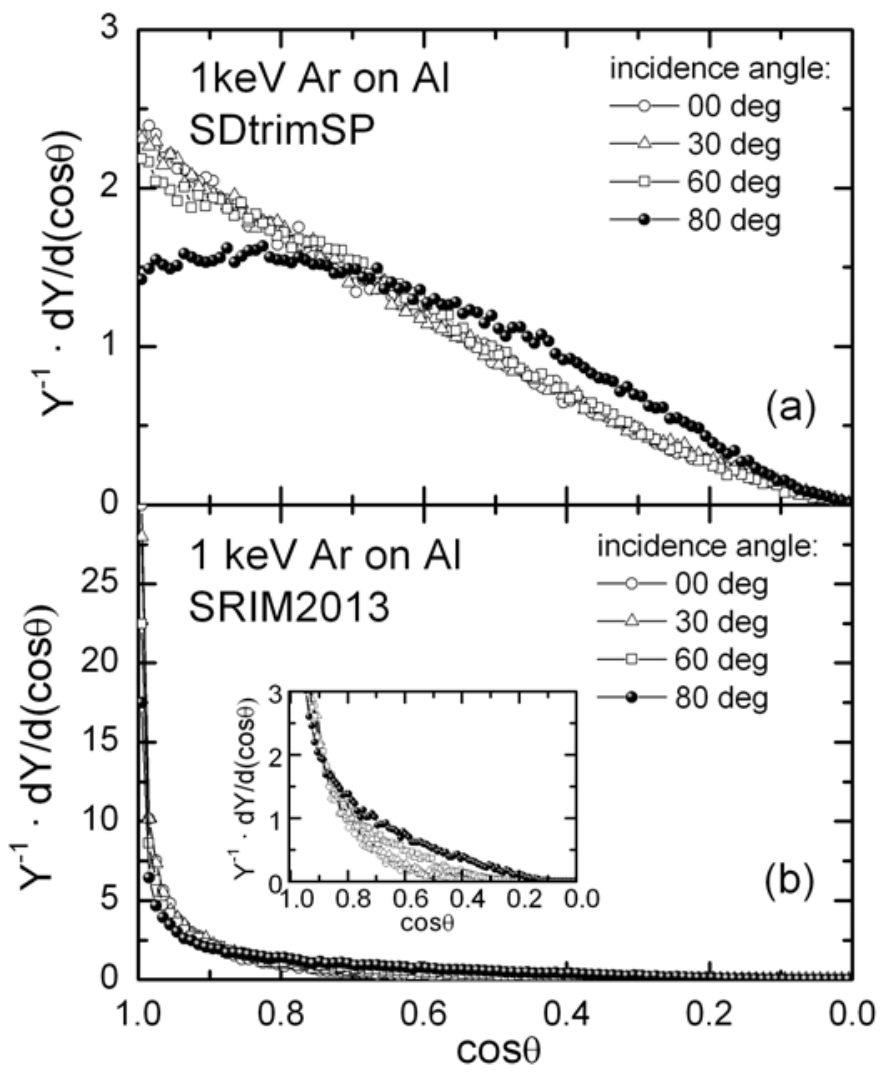

Figure 6: Normalized differential sputter yield as function of the cosine of the polar angle $\theta$ of sputtered $\mathrm{Al}$ atoms for $1 \mathrm{keV} \mathrm{Ar}$ ions and different ion incidence angles. Distributions calculated with SDTrimSP (Fig.6a) are close to a cosine distribution whereas SRIM2013 (Fig.7b) yields a distributions strongly peaked at $\cos \theta=1$, i.e. emission of sputtered atoms normal to the surface (the inset in Fig.6b has the same vertical scale as Fig.7a). 


\subsection{Sputter yield for compounds}

Several studies deal with the sputter yield measurements and simulations of compounds such as $\mathrm{SiO}_{2}$ and $\mathrm{Ta}_{2} \mathrm{O}_{5}[24,39]$. It is clear that the elemental sputtering yields are usually different (preferential sputtering of one component) and sputter erosion induces a stoichiometry change in the near surface region. SRIM as a static Monte Carlo program can only estimate sputter yields of compounds under the assumption that the initial stoichiometry is preserved. On the other hand, TRIDYN and SDTrimSP allow dynamic simulations taking into account preferential sputtering as well as stoichiometry changes within the collision cascade. For the energy dependence of the sputter yield for $\mathrm{SiO}_{2}$ and $\mathrm{Ta}_{2} \mathrm{O}_{5}$ under $\mathrm{Ar}$ ion irradiation, experimental data exist [24,39]. Here, the sputter yield was determined from the complete erosion of a thin film of several $10 \mathrm{~nm}$ thickness, corresponding to ion fluences of above about $10^{17} \mathrm{ion} / \mathrm{cm}^{2}$. Whereas reasonable agreement is obtained for $\mathrm{SiO}_{2}$ both with SRIM and SDTrimSP, there are significant deviations between SRIM simulations and experiment for $\mathrm{Ta}_{2} \mathrm{O}_{5}$ (Fig.6 of ref. [24]). SDTrimSP calculates an up to $20 \%$ smaller sputter yield (Fig.7a), In ref. [39] it is stated that the measured sputter yield is about $6 \%$ too high because of a contribution due to $\mathrm{Ar}^{2+}$ ions with high energy and thus larger sputter yield. In addition, the surface may become rough during ion erosion, which would also increase the measured sputter yield. Therefore, the SDTrimSP results are in reasonable agreement with the experimental data. In both, SRIM and SDTrimSP, simulations sputtering of $\mathrm{O}$ is the dominant process (Fig. 7b), however, only SDTrimSP run in dynamic mode gives the correct steadystate sputter yield ratio $\mathrm{Y}(\mathrm{Ta}) / \mathrm{Y}(\mathrm{O})=2 / 5$ for sputtering of $\mathrm{Ta}_{2} \mathrm{O}_{5}$. The steady state is reached after about $5 \cdot 10^{16}$ ions $/ \mathrm{cm}^{2}$ so that a comparison of the sputter yield with the experimental data can be done. Preferential sputtering of $\mathrm{O}$ leads to a strongly increased Ta concentration near the surface. Indeed, SDTrimSP reveals a high Ta concentration at the surface but also a significantly increased Ta concentration in a depth of few nm (Fig. 7c). The latter is an effect of different collision cascades of the light $\mathrm{O}$ and heavy Ta target elements. 
Since $\mathrm{SiO}_{2}$ and $\mathrm{Ta}_{2} \mathrm{O}_{5}$ both contain $\mathrm{O}$ as a light element, SRIM predicts the angular emission distribution of sputtered atoms strongly peaked along the surface normal, whereas SDTrimSP creates the expected broad cosine distribution. This is illustrated in Fig.8. The broad emission distribution from SDTrimSP corresponds to a cosine distribution, whereas from SRIM nearly all sputtered atoms would be emitted along the surface normal direction with polar angles $\beta<$ $3^{\circ}$
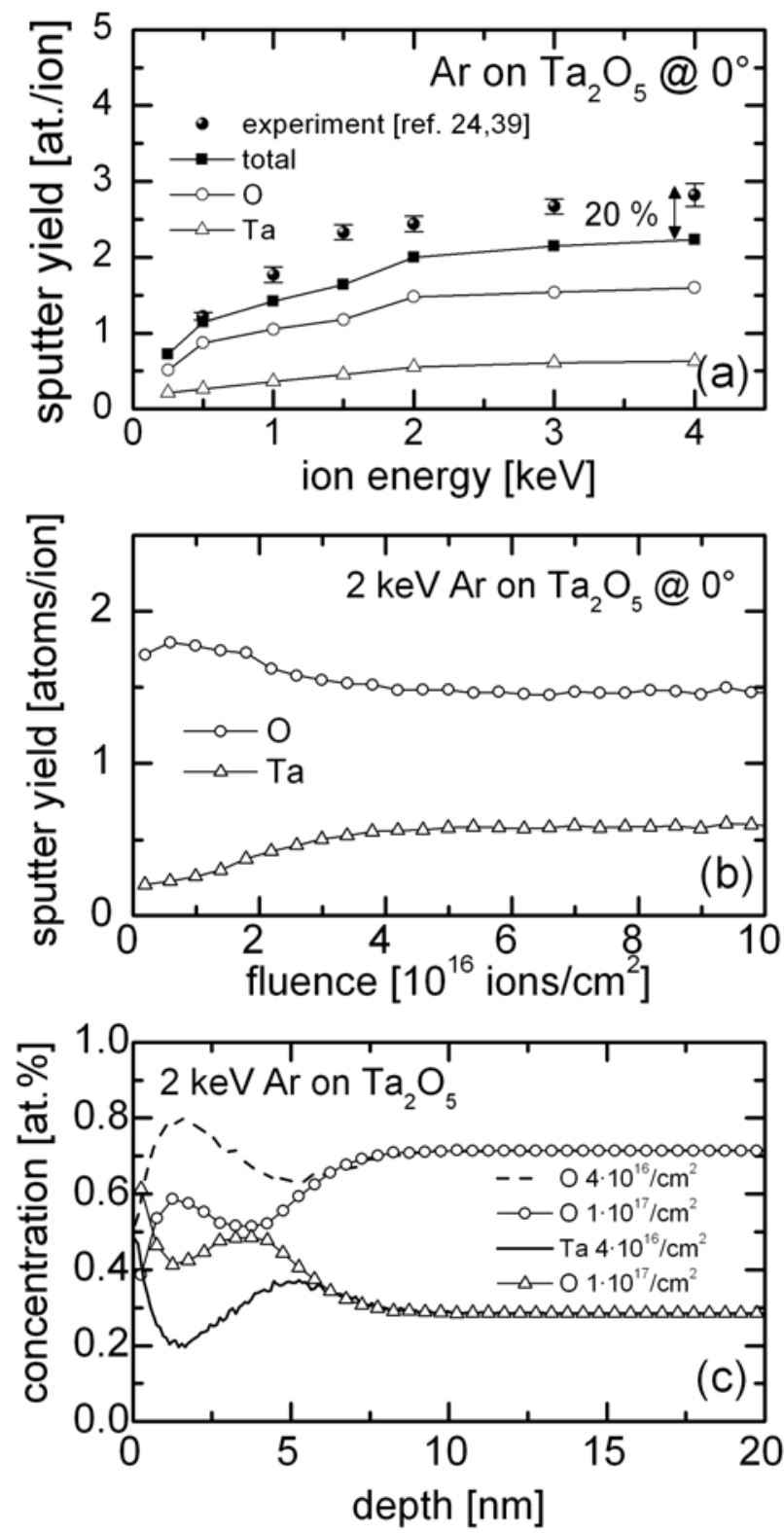

Figure 7: (a) Energy dependence of the sputter yield for Ar ions incident on a $\mathrm{Ta}_{2} \mathrm{O}_{5}$ compound target calculated with SDTrimSP. in dynamic mode for a fluence of $1 \cdot 10^{17} \mathrm{~cm}^{-2}$. The experimental data were taken from Fig.10 of ref. [39]. (b) SDTrimSP sputter yield for O and $\mathrm{Ta}$ as function of ion fluence for $2 \mathrm{keV} \mathrm{Ar}^{+}$on $\mathrm{Ta}_{2} \mathrm{O} 5$. (c) Corresponding $\mathrm{Ta}$ and $\mathrm{O}$ concentration profile after $4 \cdot 10^{16} \mathrm{Ar}^{+} / \mathrm{cm}^{2}$ and $1 \cdot 10^{17} \mathrm{Ar}^{+} / \mathrm{cm}^{2}$. 

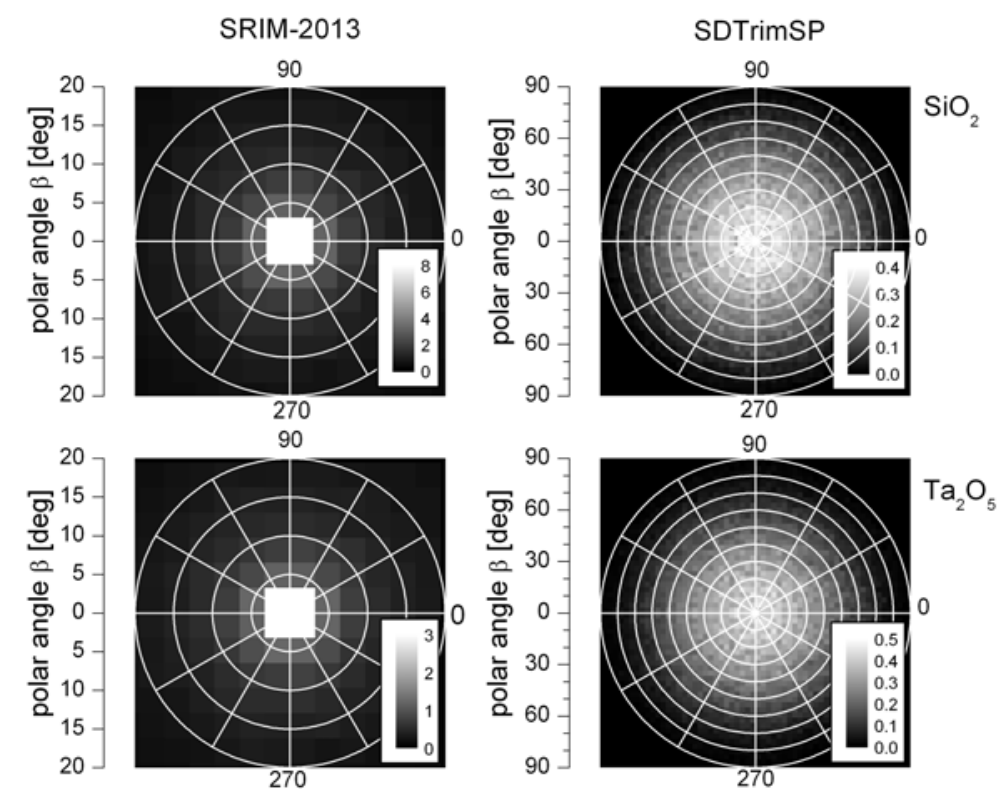

Figure 8: Stereographic projection plot of the angular distribution of sputtered atoms for 1 $\mathrm{keV}$ Ar ions at normal ion incidence on $\mathrm{SiO}_{2}$ and $\mathrm{Ta}_{2} \mathrm{O}_{5}$, calculated with SRIM-2013 and SDtrimSP. The gray scale represents the normalized differential sputter yield $\mathrm{Y}^{-1} \cdot \mathrm{dY} / \mathrm{d} \Omega$. The pixel size is $3^{\circ} \times 3^{\circ}$. According to SRIM nearly all sputtered atoms would be emitted with polar angles $\beta<3^{\circ}$.

\subsection{Sputter yield amplification effects and backscattering of ions}

Thin film growth by energetic $(100 \mathrm{eV})$ deposition of $\mathrm{Si}$ atoms on a $\mathrm{W}$ target emulates the pulsed laser deposition of Si on W, which is used to fabricate precise Si-W multilayers for Xrays Fresnel zone plate lenses [40]. The film growth rates are precisely controlled during multilayer growth and it is observed that the initial growth of Si on W is strongly retarded. Furthermore, the W/Si interface appears broadened with a decreasing concentration of $\mathrm{W}$ in Si with increasing Si layer thickness. Both observations could be explained as purely ballistic effects with the help of TRIDYN simulations [41]. The simulations were able to quantitatively reproduce the retarded growth due to the sputter yield amplification effect [42] and the broadened interface is caused by ion beam mixing. A repetition of these simulations using SDTrimSP using the $\mathrm{KrC}$ and ZBL interaction potential gave a surprising result. Only with the $\mathrm{KrC}$ potential we are able to describe the correct retarded initial film growth. The total loss of Si during growth is caused by an initial strong contribution of Si ions backscattered from W target atoms, followed by a Si sputter yield enhancement due to a 
mixed W-Si collision cascade (Fig.9). After a Si fluence of about $3 \cdot 10^{16}$ atoms $/ \mathrm{cm}^{2}$ the loss has decreased to about $10 \%$ and a constant growth rate is established. The corresponding $\mathrm{Si}$ film thickness as function of Si fluence is plotted in Fig.10. For the ZBL potential, the backscattering yield remains quite high which is due to accumulation of $\mathrm{W}$ on the surface of the growing Si film. Also there is no sputter yield amplification visible and the total loss of Si atoms remains at a high level of about $40-50 \%$ for a Si fluence up to $10^{16}$ atoms $/ \mathrm{cm}^{2}$. The Si film thickness as function of Si fluence increases almost linearly and with small growth rate. Therefore only the simulation with the $\mathrm{KrC}$ potential describes the initially retarded growth and the observed growth rates correctly. This example gives a strong indication that the ZBL potential has its limitations when it comes to low energy collisions, in particular when high Z atoms like $\mathrm{W}$ are involved. The reason probably lies in the much weaker screening of the ZBL potential at larger interaction distances compared to the $\mathrm{KrC}$ potential (see Fig.1).

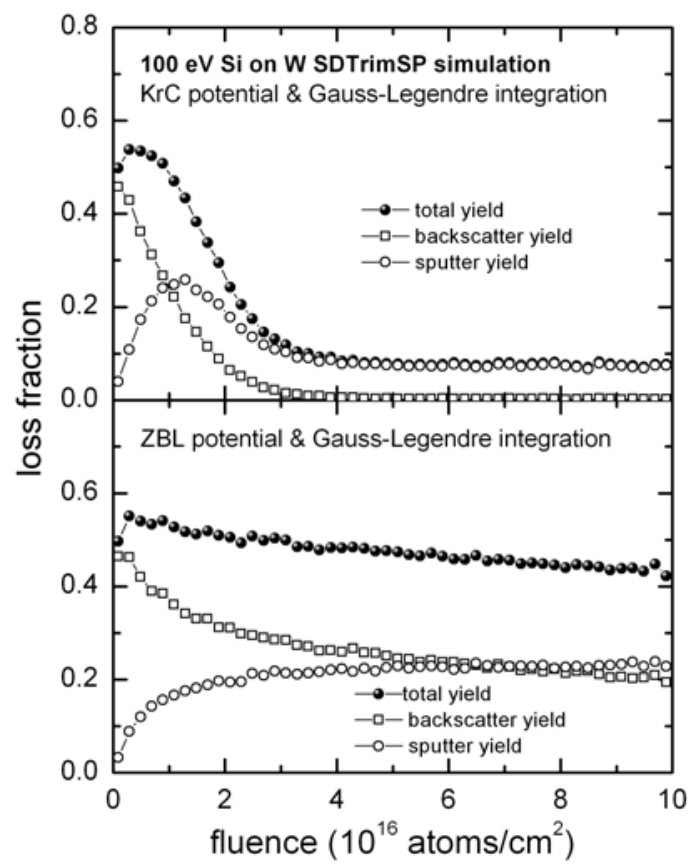

Figure 9: Calculated fraction of lost $\mathrm{Si}$ atoms either by backscattering of $\mathrm{Si}$ ions or by sputtering of Si for $100 \mathrm{eV}$ Si ions incident on a W surface. The simulation was done with SDTrimSP in dynamic mode for different interaction potentials and integration methods. For the $\mathrm{KrC}$ potential a pronounced sputter yield amplification effect occurs. For the ZBL potential the fraction of backscattered ions remains high due to accumulation of $\mathrm{W}$ at the growing surface. 


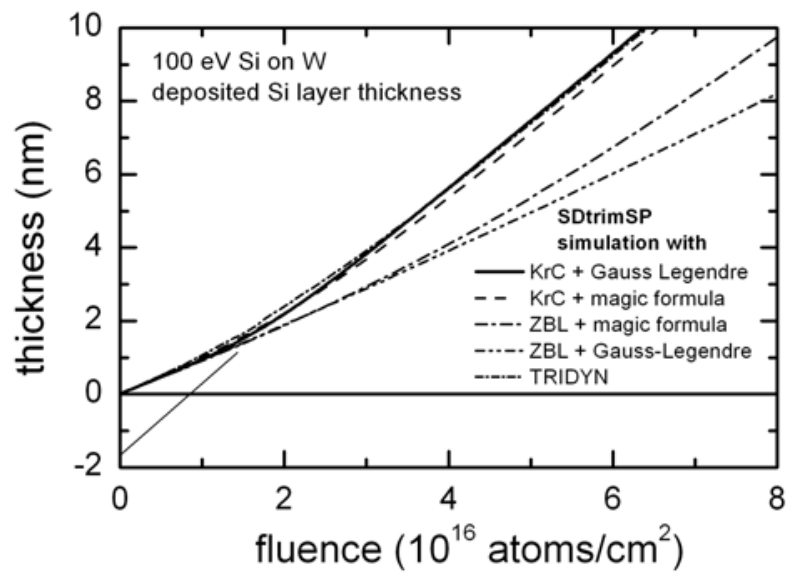

Figure 10: Layer thickness as function of ion fluence for $100 \mathrm{eV} \mathrm{Si}$ ions deposited on W, calculated with SDTrimSP and TRIDYN in dynamic mode. For SDTrimSP different interaction potentials and integration methods were chosen. Only the $\mathrm{KrC}$ potential (also used in TRIDYN) reproduces quantitatively the experimentally observed initially retarded growth rate [41].

\section{Conclusions}

SDTrimSP is a very versatile software to simulate ion sputtering also for compound targets and create detailed output on sputter yield, angular and energy distribution of sputtered atoms, concentration profiles due to preferential sputtering and collision cascade effects and sputter yield amplification effects. TRIDYN is quite comparable to SDTrimSP but offers limited input and output options. The results obtained from SDTrimSP and TRIDYN are in good quantitative agreement with many experimental sputter data. SRIM is optimized to simulate ion ranges, straggling, energy loss and damage profiles and offers a comfortable user interface. However, although SRIM calculates sputter yields and creates output data of sputter atoms, sputtering is only implemented rudimental. The comparisons with SDTrimSP and experimental data reveal severe drawbacks of SRIM. A major problem is the wrong angular distribution of sputtered atoms for targets containing low $\mathrm{Z}$ elements with $\mathrm{Z}<14$ ( $\mathrm{Z}$ $<18$ for SRIM-2008 and earlier). The angular distribution for targets elements with larger atomic number resembles a cosine distribution but is somewhat narrower compared to SDtrimSP distribution. Also the forward directed distribution in case of grazing ion incidence is not reproduced with SRIM. Another problem with SRIM is related to the angular 
dependence of the sputter yield. In particular for large angles of incidence the sputter yield is strongly overestimated. SDTrimSP allows a comparison of different interaction potentials. The example of energetic Si deposition on W reveal significant differences between the ZBL potential (used in SRIM) and the $\mathrm{KrC}$ potential (used in TRIDYN and as default in SDTrimSP). Compared to the $\mathrm{KrC}$ potential, the ZBL potential has a slowly decreasing screening function for larger interaction distances r/a $>20$ and is therefore most probably not suited to simulate low energy binary collisions.

\section{Acknowledgements}

Financial supported by the Deutsche Forschungsgemeinschaft under contract HO1125/20-1,2 is acknowledged.

\section{References}

[1] P. Sigmund, Thin Solid films 520 (2012) 6031.

[2] P. Sigmund, Phys. Rev. 184 (1969) 383.

[3] R. Behrisch (Ed.), Sputtering by particle bombardment I, Top. Appl. Phys. Vol. 47, (Springer, Heidelberg, 1981).

[4] R. Behrisch (Ed.), Sputtering by particle bombardment II, Top. Appl. Phys. Vol. 52 (Springer, Heidelberg, 1983).

[5] R. Behrisch, K. Wittmaack (Eds.), Sputtering by particle bombardment III, Top. Appl. Phys. Vol. 64 (Springer, Heidelberg, 1991).

[6] P. Sigmund (Ed.), Fundamental processes in sputtering of atoms and molecules, Mat. Fys. Medd. Dan. Vid. Selsk., volume 43, Royal Danish Academy of Sciences and Letters, Copenhagen, 1992.

[7] R. Behrisch, W. Eckstein (Eds.), Sputtering by particle bombardment, Top. Appl. Phys. Vol. 110, (Springer, Berlin, 2007). 
[8] D. Marton, Chapter 9: Film Deposition from low energy ion beams, in: Low-energy ion-surface Interactions, J.W. Rabalais, ed. (John Wiley \& Sons, 1994) p. 481-534.

[9] A. Benninghoven, F. G. Rudenauer, H. W. Werner, Secondary Ion Mass Spectrometry: Basic Concepts, Instrumental Aspects, Applications and Trends (Chemical Analysis (John Wiley \& Sons, 1987)

[10] M.M. Jakas, Phil. Trans. Roy. Soc. London A - Math. Phys. Eng. Sci., 362 (2004) 139.

[11] M.T. Robinson, Mat. Fys. Medd. Dan. Vid. Selsk. 43 (1993) 27.

[12] W. Eckstein, H.M. Urbassek, Computer simulation of the sputtering process, Volume 110 of Topics Appl. Phys. (Springer, Berlin, 2007) pp. 21-32.

[13] H. H. Andersen, Nucl. Instrum. Methods B 18 (1987) 321.

[14] W. Eckstein, Computer Simulation of Ion-Solid Interactions, (Springer,Berlin,1991)

[15] J.P. Biersack, W. Eckstein, Appl. Phys. A 34 (1984) 73.

[16] J. Ziegler, J.P. Biersack, M.D. Ziegler, SRIM-The Stopping and Ranges of Ions in Solids (SRIM Co., Chester, 2008); www.srim.org

[17] J. F. Ziegler, J.P Biersack, U. Littmark, The Stopping and Range of Ions in Solids, The Stopping and Range of Ions in Matter, Vol. 1, ed. by J.F. Ziegler (Pergamon, New York, 1985)

[18] J.P. Biersack, L.G. Haggmark, Nucl. Instrum. Methods 174 (1980) 257.

[19] W. D. Wilson, L. G. Haggmark, Phys. Rev. B15 (1977) 2458.

[20] W. Möller and W. Eckstein, Nucl. Instr. and Meth. in Phys. Res. B2 (1984) 814. (Manual TRIDYN-HZDR available at: http://www.hzdr.de/db/Cms?pOid=21578\&pNid=0)

[21] W. Möller, W. Eckstein, J.P. Biersack, Comput. Phys. Commun. 51 (1988) 355.

[22] A. Mutzke, R. Schneider, W. Eckstein, R. Dohmen, MPI for Plasma Physics, IPP Report 12/8 (2011).

[23] W. Eckstein, Sputtering yields, Vol. 110 of Topics Appl. Phys, (Springer, Berlin, 2007) pp. 33-187. 
[24] M. P. Seah and T. S. Nunney, J. Phys. D: Appl. Phys. 43 (2010) 253001.

[25] W. Eckstein, I. M. Fayazov, G. S. Knizhnik, E. S. Ma\&ova, V.A. Molchanov and A. I. Tolmachev, Nucl. Instr. Meth. B61 (1991) 12-20.

[26] R. Becerra-Acevedo, J. Bohdansky, W. Eckstein, J. Roth, NIM B 2 (1984) 631.

[27] A. P. Yalin, J. D. Williams, V. Surla and K. A. Zoerb, J. Phys. D: Appl. Phys. 40 (2007) 3194.

[28] M. Sekowski, A. Burenkov, H. Ryssel, Microelectr. Eng. 87 (2010) 1497.

[29] C. Verdeil, T. Wirtz, H.-N. Migeon, H. Scherrer, Appl. Surf. Sci. 255 (2008) 870.

[30] H. Hofsäss, O. Bobes and K. Zhang, AIP Conf. Proc. 1525 (2013) 386.

[31] A. Goehlich, N. Niemöller, and H. F. Döbele, Phys. Rev. B 62 (2000) 9349.

[32] A. Burenkov, M. Sekowski, V. Belko, H. Ryssel, Nucl. Instr. Meth B 272 (2012) 23.

[33] H. Gnaser, in R. Behrisch, W. Eckstein (Eds.), Sputtering by particle bombardment, Top. Appl. Phys., Volume 110 (Springer, Berlin, 2007) p. 231-327.

[34] S. Habenicht, W. Bolse and K.-P. Lieb, Rev. Sci. Instr. 69 (1998) 2120.

[35] TRIDYN Manual, Helmholtz Center Dresden Rossendorf, Scientific technical report FZR-317 (2002), http://www.hzdr.de/FWI/FWIT/FILES/Manual_TRIDYN_FZR.pdf

[36] S. J. Zinkle, C. Kinoshita, J. Nucl. Mat. 251 (1997) 200.

[37] G. W. Arnold, Phys. Rev. 140 (1965) A176.

[38] W. Eckstein, J.P. Biersack, Nucl. Instr. Meth B 2 (1984) 550.

[39] C. P. Hunt and M. P. Seah, Surf. And Interface Anal. 5 (1983) 199.

[40] F. Döring, A. L. Robisch, C. Eberl, M. Osterhoff, A. Ruhlandt, T. Liese, F. Schlenkrich, S. Hoffmann, M. Bartels, T. Salditt, and H. U. Krebs, Optics Express 21 (2013) 19311.

[41] C. Eberl, T. Liese, F. Schlenkrich, F. Döring, H. Hofsäss, H.-U. Krebs, Appl. Phys. A 111 (2013) 431.

[42] S. Berg, I. V. Katardjiev, J. Vac. Sci. Technol. A 17 (1999) 1916. 